\section{Yu-Chuan Li}

Center for Biomedical Informatics, Taipei Medical College, Taipei, Taiwan.

\section{Review Paper}

\section{Finding Medical Resources on the Internet}

\begin{abstract}
As in every other segment of the Internet, the amount of health-care information has increased exponentially in the past five years. Research-oriented, clinical. oriented and education-oriented medical resources have been built on the Internet by companies and institutions. Thousands of major medical web sites are currently serving millions of documents on the Internet, which are likely to double in the next 20 months, While the Internet is becoming the largest medical information repository, it is necessary that health-care professionals know efficient ways to find what they want in the vast field of medical information. This paper discusses the categorization, searching mechanisms and the impact of medical resources on the Internet.
\end{abstract}

Keywords: Internet Medical Resource, World Wide Web, Search Engine, Medical Directory Service

\section{Why the Internet}

\subsection{Size and Growth of the Internet}

The Internet has received a substantial amount of attention from the public in the past 5 years. It may mean different things to different people, but the Internet is undoubtedly one of the greatest developments that will have profound influence in the history of civilization. Since its incarnationin 1969 from the United States Advanced Research Projects Agency. (ARPA) as a 4-host computer network for military use, it had evolved into a 19,540,000-host global informationnetwork by 1997 [1]. The Internet did not attract much attention from people other than sophisticated computer users until the introduction of the WorldWide Web (WWW or the Web) in 1993 as a modality of serving multimedia and hyperlinked documents on the Internet. Because of its ease of use and versatile multimedia capability, it appealed to a much wider audience and motivated even casual computer users to use the Internet. Together with the deregulation of the Internet for commercial use, the Web gave a decisive push to the explosive growth of the Internet [2-4]. Depending on the way "Internet use" is defined, it is estimated that about 45 to 100 million users from all over the world are connected to this ubiquitous computing and communication network. More than 100 million documents, expected to be 800 million by the year 2000 , are served on the Internet that can be browsed by anyone online. These Internet documents cover an extremely wide variety of topics that are related to every aspect of human life; interesting examples including web sites that describe the experiment of cold nuclear fusion. As in every other segment of the Internet, the amount of health-care related information has increased exponentially in the past five years. The dynamic nature of the Internet will immediately make any attempt to measure the density of medical information out of date. However, akeyword search in February 1998 revealed that there were more than 930,000 documents on the Internet containing the word "cancer", 110,000 containing the text "skin cancer", and 34,000 containing "melat noma" [5]. These numbers had in creased about $30 \%$ compared to a similar search done 6 months earlier. This rate is slightly lower than the overall $70 \%$ to $120 \%$ annual growth rate of the Internet since 1993 [1].

\subsection{Unique Characteristics of the Internet}

Thanks to the ever-evolving Web Browser technology, the Web now allows presentation of almost any type of software objects on the Internet with a very easy-to-use user interface. These objects, including text, image sound, video, and animation, have enabled a new breed of medical re: sources that exist only on the Internet Medical resources on the Internet dis tinguish themselves from other infor mation distribution modalities in the they are ubiquitous, interactive, con current, easy-to-update and collectiveli searchable [6]. They are ubiquitousli, 
ilable as long as an Internet contion is present (basically, a phone ine would suffice.) They are interactive in such a way that a user can have -time feedback on her input. Many people can access the same piece of information concurrently without having to duplicate the contents and distribute them. Contents in a web site an be updated frequently without formidable cost such as reprinting a book or remaking of a CD-ROM. Moreover, all the medical information on the Internet can be searched collectively in many different ways. Current search technology allows Boolean or even natural language searches. In a Boolean search, one can combine several criteria in Boolean operators, such as AND, OR, or NOT, to accomplish a complex search. A natural language search enables a user to enter queries in everyday English. Details of Internet searching can be found in the section 3.2 .

\section{Current Status}

\subsection{Classification}

There are many ways to categorize medical resources on the Internet. For the purpose of illustrating contents of these resources, we can simply describe the contents of a medical web site as: 1) research-oriented, (2) clinical-oriented, or (3) education-oriented. The characteristics of each category are explained in the following paragraphs.

Research-oriented medical resources serve information that can aid medical researchers in different aspects of their research. For example, a research project database allows researchers to find potential collaborative partners or valuable reference projects from all over the world [7]. A gene bank or protein sequence information database enables users to find newly discovered genetic codes or protein sequences [8]. Online journals and literature databases provide researchers with a highly efficient mechanism to find and organize related publications [9].

Clinically-oriented Internet medical resources support clinicians or other health-care professionals in their daily clinical practices. For instance, a clinical practice guideline database keeps clinicians informed about the latest developments, on consensus in medical practices [10]. Online resources for drug information provide convenient access to dosing, adverse effects and contraindications of brand and generic drugs [11]. Furthermore, clinical formula calculators created by Java or other web-based languages can save physicians time in calculating various clinical parameters, such as serum osmolarity [12]. Future developments are likely to bring more complex decision-support modules, such as medical diagnostic systems, online.

Today's Internet also hosts a great wealth of medical education materials online. The low cost, associated with publishing large amounts of high-quality images on the Internet, has revolutionized the way medical education documents are presented. Medical atlases that utilize the Web platform are not limited to a handful of pictures per disease. Large online medical image databases such as the Dermatology Online Atlas from the Friedrich Alexander University, Germany and WebPath from the University of Utah are built to offer thousands of highresolution clinical images that are of great value in medical education $[13,14]$. Major multimedia teaching sites, like the Virtual Hospital from the University of Iowa, even add sounds and videos to their extensive online teaching files so that students do not just read about wheezing and crackle when learning chest auscultation, instead, they can listen to them [15].
More Continuing Medical Education (CME) courses are being offered on the Internet with better quality where physicians can earn CMEcredits online [16,17].

Even more materials can be found online that would satisfy the general public in their needs on medical information, examples include "The Merck Manual of Medical Information-Home Edition" and the Iowa Health Book $[18,19]$. The largest medical literature database, Medline, from the National Library of Medicine in the U.S., has also been opened free to all Internet users last year with an interface specifically designed for non-professional users [20]. Besides posting extensive patient education documents, some web sites go further to answer personal medical inquiries through E-mail by on-site nurses or physicians [21].

\subsection{Quantity}

The dynamic growth of medical resources on the Internet makes it difficult to estimate their quantity. However, major medical directory services such as Medical Matrix and OMNI (Organising Medical Networked Information) listed 4,072 and 2,752 medical web sites with ratings and reviews, respectively [22,23]. Most of the web sites listed here have their own domain names and contain extensive medically-related documents that fit in one or several of the categories described in Section 2.1. Some directory services take a different approach and break down their contents within a web site to fit their own systems of classification. For example, MMRL (Multimedia Medical Reference Library) listed 18,634 multimedia medical resources and Martindale's Health Science Guide ' 98 listed 44,800 teaching files and 118,000 Medical Cases [24,25]. "Medical web site" and "Internet medical resource" have been used almost interchangeably in this paper because most of the Internet 
medical resources are presented as web sites. The few resources that are not covered by the web platform include mailing lists, news groups and IRC (Internet Relay Chat) [26]. Because of the "active" or "push" (actively deliver information to your computer) nature of electronic mail, medical mailing lists still appeal to people who want discussion-style information exchange. Out of the 1,465 publicly accessible mailing lists shown on Liszt, about 120 are medical or health-related [27]. The news groups (or Usenet News) are also discussion-oriented Internet resources where any user can view all the messages posted by participants. There are more than 17,000 news groups world-wide, about 150 are medical or health-related. Unlike the asynchronous nature of mailing lists and news groups, IRC chat channels offer a mechanism for people online to have real-time chats. Very few medically-related IRC channels can be found on the enormous 17,000 channels currently available on the Internet. One reason is the "chat" and "gossip" nature of IRC seldom amounts to academic or even informative discussions that appeal to health-care professionals. For details of how mailing lists, news groups and IRC work, refer to the Liszt web site [27] .

\subsection{Quality}

The Internet has been described as a type of controlled anarchy because no central way exists to control the network [28]. This implies that there is no single way to guarantee the quality of medical information found on the Internet. The quality issue can be addressed according to the depth and accuracy of the information served. For health-care professionals, it would not be too hard to judge the depth of the contents posted by a web site if examined carefully. Since personally reviewing thousands of web sites is not practical, the best way to find high quality medical resources is by using major medical directory services and taking advantage of their rating systems. Details of these services are described in section 3.1. However, there are times when it is necessary to use Internet search engines to look for specific information. The result of a search can be thousands of hyperlinks, pointing to all kinds of resources in the world. Sometimes, even a health-care professional will not be able to tell the reliability of the information found under these circumstances. Several principles can help to improve the quality of the search:

1. Domain names are better than just IP addresses. If a site does not have a domain name like www.tmc.edu.tw but uses an IP address like 203.64.48.177, it may not be a valid and traceable site.

2. The domain name suffix GOV and EDU are better than COM. This may not always be true. But medical resources in the GOV and EDU domains have government agencies and educational institutes as backup and they are mostly notfor-profit sites. On the other hand, web sites in the COM domain are set up by companies that try to sell products. Information posted on these web sites is more-or-less for marketing purposes. However, some high-quality medical websites are in the COM domain because they profit by serving good information to health-care professionals [29].

3. Institutional web sites are better than personal web sites. There are personal web sites that provide medical information based on personal preference and belief, while institutional web sites are relatively more balanced and comprehensive. No rating systems or principles can keep up with the ever-changing Internet. Similar to the real world, the final defense of inaccurate informa- tion on the Internet would alwaye be one's own reasoning and ratio nality.

\section{Finding Medical Resources}

As mentioned in the previous sea tions, the quantity of medical resource. on the Internet is beyond anyone' capability to grasp. To make matten worse, this huge collective of inform tion continues to double every 20 months. The most efficient ways of finding what one needs in the sea of information are by; 1) referencing wif organized medical directory servical and;2) using a comprehensive Interne search engine.

\subsection{Medical Directory Services}

Medical directory services (MD, are sites that list the Internet addresser (hyperlinks) of medical resources in ar organized fashion. Different MDS. utilize different ways to categorize th hyperlink collections. For exampte Medical Matrix by Dr. Gary Malet classifies its 4,072 links into 104 categories while emphasizing its medict specialty and disease categories (whic contains 3,029 links). On the other hand, OMNI in the United Kingdort categorizes its 2,752 links into 55 cat egories [22,23]. Both MDSs have ead. of their hyperlinks reviewed with com mentaries, while the Medical Matrik also rates each link with a proprietaff 5-star rating system. Although thert are no standards for categorizing medical hyperlinks, the use of MeSH (Medf cal Subject Headings) is gaining momentum because of its clearly definet hierarchy and familiarity to reference. librarians. CliniWeb from Oregd Health Science University a Karolinska Institute MeSH Classifie Resources use only MeSH to arran their hyperlink collections, where some other MDSs are using the MeS classification as an alternative to theil 
timary categories [30,31]. Besides MDS, there are also meta-directory ervices that list and categorize sebeted directories or lists. One of the mostactively maintained medical metadirectory services is Hardin Meta Directory of Internet Health Sources from the University of Iowa [32]. If a user is familiar with certain MDS's classification system, she can quickly find what is wanted by traversing down the hierarchy or by using the on-site free-text search capability offered by most MDSs. However, if the specific information is not contained in the text of the MDS, one may need to turn to an Internet search engine that searches the Internet collectively.

\subsection{Internet Search Engines}

Laser Disks and CD-ROMs brought tous the multimediacontents that liven up esoteric medical education materials, but endless switches of disks offer little help in finding a piece of specific information hiding in stacks of CDROMs. Some may argue that the greatest advantage of bringing medical information online is that all the materials from different sources can be searched collectively [33]. Thanks to the advances in search/indexing technology and high-capacity storage devices, we are closer to being able to find anything on the Internet with one search [34]. Powerful Internet search engines such as HotBot and AltaVista have enabled users to find related medical information fast and easy, given that the right combination of keywords is known $[5,35]$. In addition to Boolean combination of keywords, state-of-the-art search functions made it possible to limit a search by date, Internet domains, country, and even by language Hsed by the target documents. AltaVista also allows natural language queries and a two-way web content translation between English and five Ather languages. Similar to the metalirectory service, there is a metatearch engine called ProFusion from
University of Arkansas that automatically forwards user queries to 9 major search engines and report an aggregated search result [36]. With the help of these search engines, users can find documents that contain almost all kinds of medical terms. For example, a search on the word "HLA-DR" revealed more than 3,000 documents that contained information about $\mathrm{Hu}$ man Lymphocyte Antigen DR typing. This kind of searching capability, combined with the exponential growth of medical information online, will soon make the Internet the largest medical knowledge repository in history.

\section{The Future}

The Internet has experienced explosive growth in the past five years. For money or fame, companies and educational institutions have been substantially investing in the Internet with a growing number of net citizens (or netizens) in mind. We can be sure that the quantity of medical information will keep increasing significantly in response to the market that is clearly rising. However, whether the quality will follow remains to be seen.

The progressive traffic congestion of the Internet due to its growth is considered one of the most serious threats to the continuing development of the Internet itself. To resolve the problem, Internet2, the Next-Generation Internet (NGI) and the STAR TAP project were proposed and experimented in countries world-wide [37-39]. Together with the advances of Internet software technology, we will have more bandwidth, more interactivity andmore multimedia online in the next five years. Consequently, more types of medical resources and services will be implemented on the Internet, which can benefit patients and health-care providers alike. Possible examples include medical consul- tations online where medical specialists can perform consultations through videoconferences and electronic patient records; outpatient visits through a virtual clinic on the Internet; webbased home care, and virtual pharmacies where prescriptions can be filled online.

The growth of Internet medical resources will significantly influence the behavior of both health-care professionals and the general public [40]. With the rich resources discussed in the Sections 3.1 and 3.2, patients and health-care professionals now have extremely flexible ways to access documents and databases that they could never have had otherwise. The impact is profound and long lasting. Patients of the Internet generation will have easy access to available treatment options and background knowledge of their own diseases before they visit a physician. On the other hand, the physician will have more comprehensive and updated information when seeing a patient. With the help of high-quality Internet medical resources, patients and caregivers of the future would have useful medical information at their fingertips.

\section{References}

1. Network Wizard. Internet domain survey. URL: http://www.nw,com/.

2. History of the Internet. URL: http:// www.isoc.org/internet-history/.

3. Pallen M. Guide to the internet. The world wide web. Brit Med J 1995;311:1552-6.

4. Pallen $\mathbf{M}$. Introducing the internet. Brit Med J 1995;311:1422-4.

5. HotBot. URL: http://www.hotbot.com/.

6. Medical Informatics in Taiwan. Biomedical information server. URL: http://li.tmc. edu.tw/.

7. Community of Science. Funded research. URL:http://medoc.gdb.org/.

8. National Center for Biotechnology Information. URL: http://www.ncbi. nlm.nih.gov/.

9. National Library of Medicine (NLM). Free MEDLINE: PubMed and Internet Grateful Med. URL: http://www.nlm.nih.gov/ databases/freemedl.html. 
10. Agency for Health Care Policy and Research (AHCPR). Clinical practice guidelines online. URL:http://www.ahcpr.gov/ clinic/

11. MedEc Interactive-The medical center of the interactive world. URL: http:// www.medecinteractive.com/.

12. MedStudent's Homepage. Clinical calculators online. URL: http:// www.medstudents.com.br/calculat/ index 2 .htm.

13. Dermatology Online Atlas (DOIA). URL: http://www.derma.med.uni-erlangen,de/ bilddb/index_e.htm.

14. Spencer S. Eccles Health Sciences Library. The internet pathology laboratory for medical education. URL: http://wwwmedlib.med.utah.edu/WebPath/ webpath.html.

15. University of Iowa. The virtual hospital. URL: http://www.vh,org/.

16. National Library of Medicine, Health Services/ Technology Assessment Text (HSTAT). Continuing education - NIH consensus development program. URL: http://text.nlm.nih.gov/nih/upload-v3/ Continuing_Education/cme.html.

17. CMEWeb - The physician's one-stop resource for CME on the web. URL: http:// www.cmeweb.com.

18. Merck Publications. The Merck manuals. URL: http://www.merck.com/pubs/.

19. University of Iowa. Virtual Hospital: Iowa Health Book. URL: http://vh.radiology. uiowa.edu/Patients/IowaHealthBook/
PatientInfo,html.

20. The National Center for Biotechnology Information. PubMed. URL: http:// www.ncbi.nlm.nih.gov/PubMed/.

21. Health Inquiry Network. URL: http:// health.seed.net.tw/.

22. Medical Matrix, RL: http://www. medmatrix.org/index.asp?

23. OMNI (Organising Medical Networked Information). URL: http://omni.ac.uk/.

24. Jonathan Tward's Multimedia Medical Reference Library (MMRL), a virtual library. URL: http://www.med-library.com/ medlibrary/.

25. Martindale's Health Science Guide - '98. URL: http://www-sci.lib.uci.edu/HSG/ HSGuide.html.

26. Kramer JM. Cath A. Medical resources and the internet. Making the connection. Arch Intern Med 1996;8:833-42.

27. Listz, the mailing list directory. URL:http:/ /www.liszt.com/.

28. Wang KK., Song WK. The physician and the internet. Mayo Clinic Proceedings 1997; 1:66-71.

29. Medscape. URL: http://www.medscape. $\mathrm{com} /$.

30. Oregon Health Sciences University. CliniWeb. URL: http://www.ohsu.edu/ cliniweb/.

31. Nyheter Fran Karolinska Institutets Bibliotek. Diseases, disorders and related topics. URL: http://www.mic.ki.se/Diseases/index.html

32. University Of Iowa Libraries. Hardin meta directory of internet health sources. URe. http://www.lib.uiowa.edu/hardin/m index.html.

33. Schatz BR. Information retrieval in digio libraries: bringing search to the net. Scier 1997;5298:327-34

34. Seltzer R, Ray EJ, Ray DS. How to Find Anything on the Internet. The Alta Vi Search Revolution. Osborne: McGraw-k 1996.

35. Digital Equipment Corporation. AltaVis URL: http://www.altavista.digital.co

36. DesignLab - ProFusion. URL: http www.designlab.ukans.edu/profusiond

37. University Corporation for Advane Internet Development. Internet2 Hom URL: http://www.internet2.edu.

38. Next Generation Internet (NGI) Initiativ URL: http://www.ngi.gov/.

39. Star Tap - major funding provided by the national science foundation. URL: http: www.startap.net/.

40. Huang MP, Alessi NE. The Internet and future of psychiatry. Am J Psychi 1996;7:861-9.

Address of the author:

Yu-Chuan Li, M.D., Ph.D.,

Center for Biomedical Informatics,

Taipei Medical College,

Tapei, Taiwan.

Phone: +8862377.6730

Fax: +8862 7339049

e-mail:jack@tmc.edu.tw 